\title{
Automated Algorithm Configuration and Parameter Tuning
}

\author{
Holger H. Hoos
}

\section{Introduction}

Computationally challenging problems arise in the context of many applications, and the ability to solve these as efficiently as possible is of great practical, and often also economical importance. Examples of such problems include scheduling, timetabling, resource allocation, production planning and optimisation, computer-aided design and software verification. Many of these problems are $\mathscr{N} \mathscr{P}$-hard and considered computationally intractable, because there is no polynomial-time algorithm that can find solutions in the worst case (unless $\mathscr{N} \mathscr{P}=\mathscr{P}$ ). However, by using carefully crafted heuristic techniques, it is often possible to solve practically relevant instances of these 'intractable' problems surprisingly effectively (see, e.g., Prasad et al, 2005; Applegate et al, 2006; Pop et al, 2002). ${ }^{1}$

The practically observed efficacy of these heuristic mechanisms remains typically inaccessible to the analytical techniques used for proving theoretical complexity results, and therefore needs to be established empirically, on the basis of carefully designed computational experiments. In many cases, state-of-the-art performance is achieved using several heuristic mechanisms that interact in complex, non-intuitive ways. For example, a DPLL-style complete solver for SAT (a prototypical $\mathscr{N} \mathscr{P}$ complete problem with important applications in the design of reliable soft- and hardware) may use different heuristics for selecting variables to be instantiated and the values first explored for these variables, as well as heuristic mechanisms for managing and using logical constraints derived from failed solution attempts. The activation, interaction and precise behaviour of those mechanisms is often controlled by parameters, and the settings of such parameters have a substantial impact on the

Holger H. Hoos

Department of Computer Science, University of British Columbia, 2366 Main Mall, Vancouver, BC, V6T 1Z4, Canada, e-mail: hoos@cs.ubc.ca

${ }^{1}$ We note that the use of heuristic techniques does not imply that the resulting algorithms are necessarily incomplete or do not have provable performance guarantees, but often results in empirical performance far better than the bounds guaranteed by rigorous theoretical analysis. 
efficacy with which a heuristic algorithm solves a given problem instance or class of problem instances. For example, the run-time of CPLEX 12.1 - a widely used, commercial solver for mixed integer programming problems - has recently been demonstrated to vary up to a factor of over 50 with the settings of 76 user-accessible parameters (Hutter et al, 2010c).

A problem routinely encountered by designers as well as end-users of parameterised algorithms is that of finding parameter settings (or configurations) for which the empirical performance on a given set of problem instances is optimised. Formally, this algorithm configuration or parameter tuning problem can be stated as follows:

Given

- an algorithm $A$ with parameters $p_{1}, \ldots, p_{k}$ that affect its behaviour,

- a space $C$ of configurations (i.e., parameter settings), where each configuration $c \in C$ specifies values for $A$ 's parameters such that $A$ 's behaviour on a given problem instance is completely specified (up to possible randomisation of $A$ ),

- a set of problem instances $I$,

- a performance metric $m$ that measures the performance of $A$, on instance set $I$ for a given configuration $c$,

find a configuration $c^{*} \in C$ that results in optimal performance of $A$ on $I$ according to metric $m$.

In the context of this problem, the algorithm whose performance is to be optimised is often called the target algorithm, and we use $A(c)$ to denote target algorithm $A$ under a specific configuration $c$. The set of values any given parameter $p$ can take is called the domain of $p$. Depending on the given target algorithm, various types of parameters may occur. Categorical parameters have a finite, unordered set of discrete values; they are often used to select between a number of alternative mechanisms or components. Using Boolean parameters, heuristic mechanism can be activated or deactived, while the behaviour and interaction of these mechanisms is often controlled by integer- and real-valued parameters (the former of which are a special cases of ordinal parameters, whose domains are discrete and ordered). Conditional parameters are only active when other parameters are set to particular values; they routinely arise in the context of mechanisms that are activated or selected using some parameter, and whose behaviour is then controlled by other parameters (where the latter parameters conditionally depend on the former). Sometimes, it is useful to place additional constraints on configurations, e.g., to exclude certain combinations of parameter values that would lead to ill-defined, incorrect or otherwise undesirable behaviour of a given target algorithm.

Clearly, the number and types of parameters, along with the occurrence of conditional parameters and constraints on configurations, determine the nature of the configuration space $C$ and have profound implications on the methods to be used for searching performance-optimising configurations within that space. These meth- 
ods range from well-known numerical optimisation procedures, such as the NelderMead Simplex algorithm (Nelder and Mead, 1965; Búrmen et al, 2006) or the more recent, gradient-free CMA-ES algorithm (Hansen, 2006; Hansen and Ostermeier, 2001; Hansen and Kern, 2004), to approaches based on experimental design methods (see, e.g., Birattari et al, 2002; Balaprakash et al, 2007; Adenso-Diaz and Laguna, 2006), response-surface models (see, e.g., Jones et al, 1998; Bartz-Beielstein, 2006) or stochastic local search procedures (see, e.g., Hutter et al, 2007b, 2009a).

In general, when configuring a specific target algorithm, it is desirable to find parameter configurations that work well on problem instances other than those in the given instance set $I$. To this end, care needs to be taken in selecting the instances in $I$ to be representative of the kinds of instances to which the optimised target algorithm configuration is expected to be applied. Difficulties can arise when $I$ is small, yet contains very different types of instances. To recognise situations in which a configured target algorithm, $A\left(c^{*}\right)$, fails to perform well when applied to instances other than those used in the configuration process, it is advisable to test it on a set of instances not contained in $I$; this can be done by including in $I$ only part of the overall set of instances available, or by means of cross validation.

It is also advisable to investigate performance variation of $A\left(c^{*}\right)$ over instance set $I$, since, depending on the performance metric $m$ used for the configuration of $A$ and differences between instances in $I$, the optimised configuration $c^{*}$ might represent a tradeoff between strong performance on some instances at the cost of weaker performance on others. In particular, when using robust statistics, such as median run-time, as a performance metric, detrimental performance on large parts of a given instance set can result. To deal effectively with target algorithm runs in which no solution was produced (in particular, time-outs encountered when optimising runtime), it is often useful to use a performance metric based on penalised averaging, in which a fixed penalty is assigned to any unsuccessful run of $A$ (see also Hutter et al, 2009a).

In the existing literature, the terms algorithm configuration and parameter tuning are often used interchangeably. We prefer to use parameter tuning in the context of target algorithms with relatively few parameters with mostly real-valued domains, and algorithm configuration in the context of target algorithms with many categorical parameters. Following Hoos (2008), we note that algorithm configuration problems arise when dealing with an algorithm schema that contains a number of instantiable components (typically, subprocedures or functions), along with a discrete set of concrete choices for each of these. While most standard numerical optimisation methods are not applicable to these types of algorithm configuration problems, FRace (Birattari et al, 2002; Balaprakash et al, 2007), Calibra (Adenso-Diaz and Laguna, 2006) and ParamILS (Hutter et al, 2007b, 2009a) have been used successfully in this context. However, so far only ParamILS has been demonstrated to be able to deal with the vast design spaces resulting from schemata with many, independently instantiable components (see, e.g., KhudaBukhsh et al, 2009; Tompkins and Hoos, 2010), and promising results have been achieved by a genetic programming procedure applied to the configuration of local search algorithms for SAT (Fukunaga, 
2002, 2004), as well as by a recent gender-based genetic algorithm (Ansótegui et al, 2009).

In the remainder of this chapter, we discuss three classes of methods for solving algorithm configuration and parameter tuning problems. Racing procedures iteratively evaluate target algorithm configurations on problem instances from a given set and use statistical hypothesis tests to eliminate candidate configurations that are significantly outperformed by other configurations; ParamILS uses a powerful stochastic local search (SLS) method to search within potentially vast spaces of candidate configurations of a given algorithm; and sequential model-based optimisation (SMBO) methods build a response surface model that relates parameter settings to performance, and use this model to iteratively identify promising settings. We also give a brief overview of other algorithm configuration and parameter tuning procedures and comment on the applications in which various methods have proven to be effective.

While we deliberately limit the scope of our discussion to the algorithm configuration problem defined earlier in this section, we note that there are several conceptually closely related problems that arise in the computer-aided design of algorithms (see also Hoos, 2008): per-instance algorithm selection methods choose one of several target algorithms to be applied to a given problem instance based on properties of that instance determined just before attempting to solve it (see, e.g., Rice, 1976; Leyton-Brown et al, 2003; Guerri and Milano, 2004; Xu et al, 2007, 2008); similarly, per-instance algorithm configuration methods use instance properties to determine the specific configuration of a parameterised target algorithm to be used for solving a given instance (see, e.g., Hutter et al, 2006). Reactive search procedures, on-line algorithm control methods and adaptive operator selection techniques switch between different algorithms, heuristic mechanisms or parameter configurations while running on a given problem instance (see, e.g., Carchrae and Beck, 2005; Battiti et al, 2008; Da Costa et al, 2008); and dynamic algorithm portfolio approaches repeatedly adjust the allocation of CPU shares between algorithms that are running concurrently on a given problem instance (see, e.g., Gagliolo and Schmidhuber, 2006).

Furthermore, we attempt neither to cover all algorithm configuration methods that can be found in the literature, nor to present all details of the procedures we describe; instead, we focus on three fundamental approaches of algorithm configuration methods and survey a number of prominent methods based on these, including the state-of-the-art procedures at the time of this writing. We briefly discuss selected applications of these procedures to illustrate their scope and performance, but we do not attempt to give a complete or detailed account of the empirical results found in the literature. 


\section{Racing Procedures}

Given a number of candidate solvers for a given problem, the concept of racing is based on a simple, yet compelling idea: sequentially evaluate the candidates on a series of benchmark instances and eliminate solvers as soon as they have fallen too far behind the current leader, i.e., the candidate with the overall best performance at a given stage of the race.

Racing procedures have been originally introduced for solving model selection problems in machine learning. The first such technique, dubbed Hoeffding Races (Maron and Moore, 1994), was introduced in a supervised learning scenario, where a black-box learner is evaluated by measuring its error on a set of test instances. The key idea is to test a given set of models, one test instance at a time, and to discard models as soon as they are shown to perform significantly worse than the best ones. Performance is measured as error over all test instances evaluated so far, and models are eliminated from the race using non-parametric bounds on the true error, determined based on Hoeffding's inequality (which gives an upper bound on the probability for the sum of random variables to deviate from its expected value). More precisely, a model is discarded from the race, if the lower bound on its true error (for a given confidence level $1-\delta$ ) is worse than the upper bound on the error of the currently best model. As a result, the computational effort expended in evaluating models becomes increasingly focussed on promising candidates, and the best candidate models end up getting evaluated most thoroughly.

This idea can be easily transferred to the problem of selecting an algorithm from a set of candidates, where each candidate may correspond to a configuration of a parameterised algorithm (Birattari et al, 2002). In this context, candidate algorithms (or configurations) are evaluated on a given set of problem instances. As in the case of model selection, the race proceeds in steps, where in each step, every candidate is evaluated on the same instance, taken from the given instance set, and candidates that performed significantly worse on the instances considered so far are eliminated from the race. (We note that the evaluation of candidates in each step can, in principle, be performed independently in parallel.)

This procedure requires that the set of candidate algorithms be finite and, since in the initial steps of a race all candidates will need to be evaluated, of somewhat reasonable size. Therefore, when applied to algorithm configuration or parameter tuning scenarios with continuous parameters, racing approaches need to make use of discretisation or sampling techniques. In the simplest case, all continuous parameters are discretised prior to starting the race. Alternatively, stages of sampling and racing can be interleaved, such that the candidate configurations being considered become increasingly concentrated around the best performing configurations.

In the following, we will first present the F-Race procedure of Birattari et al (2002) in more detail and outline its limitations. We will then discuss variations of F-Race that overcome those weaknesses (Balaprakash et al, 2007; Birattari et al, 2010), and finally summarise some results achieved by these racing procedures in various algorithm configuration scenarios. 


\subsection{F-Race}

The F-Race algorithm by Birattari et al (2002) closely follows the previously discussed racing procedure. Similar to Hoeffding races, it uses a non-parametric test as the basis for deciding which configurations to eliminate in any given step. However, rather than just performing pairwise comparisons with the currently best configuration (the so-called incumbent), F-Race first uses the rank-based Friedman test (also know as Friedman two-way analysis of variance by ranks) for $n i$ independent $s$ variate random variables, where $s$ is the number of configurations still in the race, and $n i$ is the number of problem instances evaluated so far. The Friedman test assesses whether the $s$ configurations show no significant performance differences on the $n i$ given instances; if this null hypothesis is rejected, i.e., if there is evidence that some configurations perform better than others, a series of pairwise post hoc tests between the incumbent and all other configurations is performed. All configurations found to have performed significantly worse than the incumbent are eliminated from the race. An outline of the F-Race procedure for algorithm configuration, as introduced by Birattari et al, 2002, is shown in Fig. 1; as mentioned by Balaprakash et al, 2007, runs on a fixed number of instances are performed before the Friedman test is first applied. The procedure is typically terminated either when only one configuration remains, or when a user-defined time budget has been exhausted.

The Friedman test involves ranking the performance results of each configuration on a given problem instance; in the case of ties, the average of the ranks that would have been assigned without ties is assigned to each tied value. The test then determines whether some configurations tend to be ranked better than others when considering the rankings for all instances considered in the race up to the given iteration. Following Birattari et al (2002), we note that performing the ranking separately for each problem instance amounts to a blocking strategy on instances. The use of this strategy effectively reduces the impact of noise effects that may arise from the performance variation observed over the given instances set for any configuration of the target algorithm under consideration; this can become critical when those performance variations are large, as has been observed for many algorithms for various hard combinatorial problems (see, e.g., Gomes et al, 2000; Hoos and Stützle, 2000).

\subsection{Sampling F-Race and Iterative F-Race}

A major limitation of this basic version of F-Race stems from the fact that in the initial steps, all given configurations have to be evaluated. This property of basic F-Race severely limits the size of the configuration spaces to which the procedure can be applied effectively - particularly, when dealing with configuration spaces corresponding to so-called full factorial designs, which contain all combinations of values for a set of discrete (or discretised) parameters. Two more recent variants of F-Race, Sampling F-Race and Iterative F-Race, have been introduced to address 


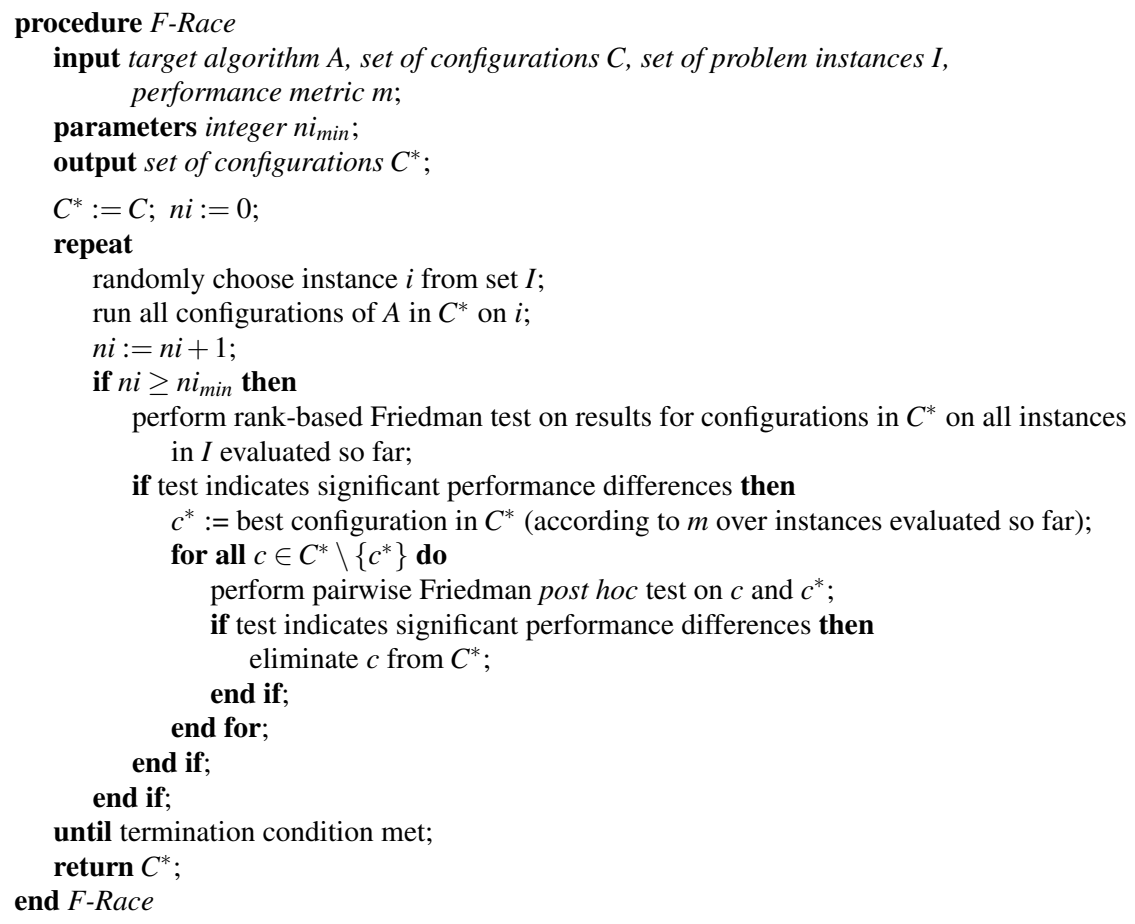

Fig. 1 Outline of F-Race for algorithm configuration (original version, according to Birattari et al, 2002). In typical applications, $n i_{\min }$ is set to values between 2 and 5 ; further details are explained in the text. When used on its own, the procedure would typically be modified to return $c^{*} \in C^{*}$ with the best performance (according to $m$ ) over all instances evaluated within the race.

this limitation (Balaprakash et al, 2007); both use the previously described F-Race procedure as a subroutine.

Sampling F-Race (short: RSD/F-Race) is based on the idea of using a sampling process to determine the initial set of configurations subsequently used in a standard F-Race. In RSD/F-Race, a fixed number $r$ of samples is determined using a socalled Random Sampling Design, in which each configuration is drawn uniformly at random from the given configuration space $C$. (In the simplest case, where no conditional parameters or forbidden configurations exist, this can be done by sampling values for each parameter independently and uniformly at random from the respective domain.) As noted by Balaprakash et al (2007), the performance of this procedure depends substantially on $r$, the number of configurations sampled, in relation to the size of the given configuration space.

A somewhat more effective approach for focussing a procedure based on F-Race on promising configurations is Iterative F-Race (short: I/F-Race). The key idea behind I/F-Race is the use of an iterative process, where in the first stage of each 


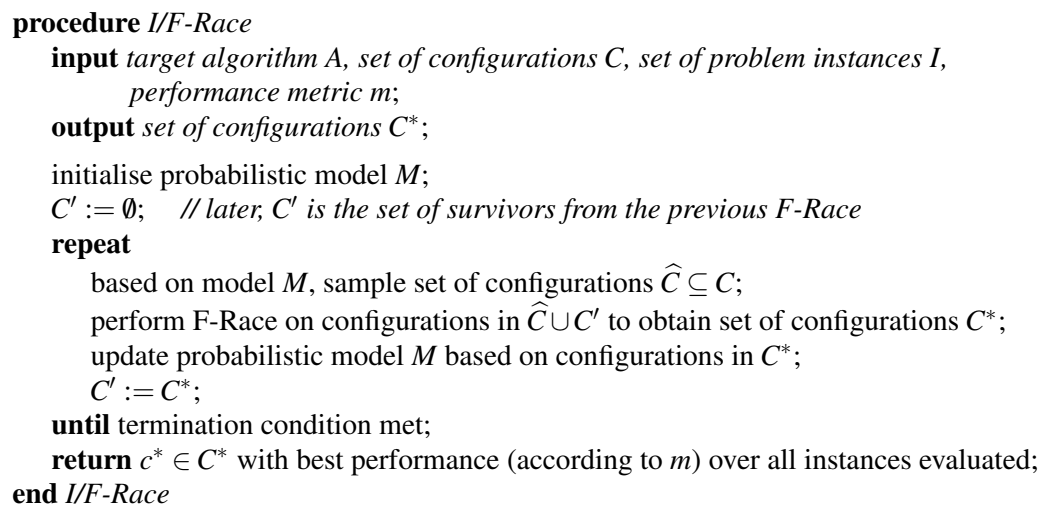

Fig. 2 High-level outline of Iterated F-Race, as introduced by Balaprakash et al, 2007; details are explained in the text. The most recent version of I/F-Race slightly deviates from this outline (see Birattari et al, 2010).

iteration, configurations are sampled from a probabilistic model $M$, while in the second stage, a standard F-Race is performed on the resulting sample, and the configurations surviving this race are used to define or update the model $M$ used in the following iteration. (See Fig. 2.)

The probabilistic model used in each iteration of I/F-Race consists of a series of probability distributions, $\mathscr{D}_{1}, \ldots, \mathscr{D}_{s}$, each of which is associated with one of $s$ 'promising' parameter configurations, $c_{1}, \ldots, c_{s}$. Balaprakash et al (2007) consider only numerical parameters and define each distribution $\mathscr{D}_{i}$ to be a $k$-variate normal distribution $\mathscr{N}_{i}:=\mathscr{N}\left(\mu_{i}, \Sigma_{i}\right)$ that is centred on configuration $c_{i}$, i.e., $\mu_{i}=c_{i}$. They further define the covariance between any two different parameters in a given $\mathscr{N}_{i}$ to be zero, such that $\mathscr{N}_{i}$ can be factored into $k$ independent, univariate normal distributions. To start the process with an unbiased probabilistic model, in the first iteration of I/F-Race, a single $k$-variate uniform distribution is used, which is defined as the product of the $k$ independent uniform distributions over the ranges of each given parameter (we note that this can be seen as a degenerate case of the normal distributions used subsequently, in which the variance is infinite and truncation is applied).

In each iteration of I/F-Race, a certain number of configurations are sampled from the distributions $\mathscr{N}_{1}, \ldots, \mathscr{N}_{s}$. In the first iteration, this corresponds to sampling configurations uniformly at random from the given configuration space. In subsequent iterations, for each configuration to be sampled, first, one of the $\mathscr{N}_{i}$ is chosen using a rank-based probabilistic selection scheme based on the performance of the configuration $c_{i}$ associated with $\mathscr{N}_{i}$ (for details, see Balaprakash et al, 2007), and then, a configuration is sampled from this distribution. Values that are outside the range allowable for a given parameter are set to the closer of the two boundaries, and settings for parameters with integer domains are rounded to the nearest valid value. The number $a$ of configurations sampled in each iteration depends on the number 
$s$ of configurations that survived the F-Race in the previous iteration; Balaprakash et al (2007) keep the overall number of configurations considered in each iteration of I/F-Race constant at some value $r$, and therefore simply replace those configurations eliminated by F-Race with newly sampled ones (i.e., $a:=r-s$, where in the first iteration, $s=0$ ).

The resulting population of $a+s$ configurations is subjected to a standard FRace; this race is terminated using a complex, disjunctive termination condition that involves a (lower) threshold on the number of surviving configurations as well as upper bounds on the computational budget (measured in target algorithm runs) and the number of problem instances considered. ${ }^{2}$ Each of the F-races conducted within I/F-Race uses a random permutation of the given instance set, in order to avoid bias due to a particular instance ordering. The $s$ configurations that survived the race (where the value of $s$ depends on the part of the termination condition that determined the end of that race) induce the probabilistic model used in the following iteration of I/F-Race.

To increasingly focus the sampling process towards the most promising configurations, the standard deviations of the component distributions of the probabilistic models $\mathscr{N}_{i}$ are gradually decreased using a volume reduction technique. More precisely, after each iteration, the standard deviation vector $\sigma_{i}$ of each distribution $\mathscr{N}_{i}$ is scaled by a factor $(1 / r)^{k}$, where $r$ is the total number of configurations entered into the F-Race, and $k$ is the number of given parameters; this corresponds to a reduction of the total volume of the region bounded by $\mu_{i} \pm \sigma_{i}$ (over all $k$ parameters) by a factor of $r$. At the beginning of I/F-Race, when configurations are sampled uniformly, the standard deviation values are (somewhat arbitrarily) set to half of the range of the respective parameter values.

I/F-Race, as specified by Balaprakash et al (2007), assumes that all parameters are numerical. This limitation is overcome in a later variant (Birattari et al, 2010), which supports categorical parameters by sampling their values from discrete probability distributions that are updated by redistributing probability mass to values seen in good configurations, as determined by F-Race. This version of I/F-Race, which we call I/F-Race-10 for clarity, also differs from the one described previously in several other aspects. Notably, the number of iterations in I/F-Race-10 is determined as $2+\left\lfloor\log _{2}(k)+0.5\right\rfloor$, and the overall computational budget (i.e., number of target algorithm runs) is distributed equally over these iterations. Furthermore, the number $r$ of configurations considered at iteration number $t$ is set to $\lfloor b /(5+t)\rfloor$, where $b$ is the computational budget available for that iteration; this leads to fewer configurations being considered in later iterations. The threshold on the number of survivors below which any given F-Race is terminated is also determined as $2+\left\lfloor\log _{2}(k)+0.5\right\rfloor$. Finally, I/F-Race-10 handles conditional parameters by only sampling values for them when they are active, and by only updating the respective component of the model in situations where such parameters are active in a configuration surviving one of the subsidiary F-Races. (For further details, see Birattari et al, 2010.)

2 The threshold mechanism ends the race as soon as the number of survivors has fallen below $k$, the number of target algorithm parameters. 


\subsection{Applications}

Balaprakash et al (2007) describe applications of F-Race, Sampling F-Race and Iterative F-Race to three high-performance stochastic local search algorithms: MAXMIN Ant System for the TSP with 6 parameters (Stützle and Hoos, 2000), an estimation-based local search algorithm for the probabilistic TSP (PTSP) with 3 parameters (Balaprakash et al, 2010), and a simulated annealing algorithm for vehicle routing with stochastic demands (VRP-SD) with 4 parameters (Pellegrini and Birattari, 2006). The empirical results from these case studies indicate that both, Sampling F-Race and Iterative F-Race can find good configurations in spaces that are too big be handled effectively by F-Race, and that Iterative F-Race tends to give better results than Sampling F-Race, especially when applied to more difficult configuration problems. Both, the PTSP and the VRP-SD algorithms as configured by Iterative F-Race represented the state of the art in solving these problems at the time of this study.

Further applications of F-Race have recently been summarised by Birattari et al (2010). These include tuning the parameters of various meta-heuristic algorithms for university timetabling problems (Rossi-Doria et al, 2003), of a control system for simple robots (Nouyan et al, 2008), and of a new state-of-the-art memetic algorithm for the linear ordering problem (Schiavinotto and Stützle, 2004). In all of these cases, the basic F-Race algorithm was applied to target algorithms with few parameters and rather small configuration spaces (48-144 configurations).

Yuan et al (2008) report an application of I/F-Race for tuning various heuristic algorithms for solving a locomotive scheduling problem provided by the German railway company, Deutsche Bahn. The target algorithms considered in this work had up to 5 parameters, mostly with continuous domains. The most complex application of I/F-Race reported by Birattari et al (2010) involves 12 parameters of the ACOTSP software, some of which conditionally depend on the value of others.

While these (and other) racing procedures have been demonstrated to be useful for accomplishing a broad range of parameter tuning tasks, it is somewhat unclear how well they perform when applied to target algorithms with many more parameters, and how effectively they can deal with the many categorical and conditional parameters arising in the context of more complex computer-aided algorithm design tasks, such as the ones considered by Hutter et al (2007a), KhudaBukhsh et al (2009), Hutter et al (2010c), as well as by Tompkins and Hoos (2010).

\section{ParamILS}

When manually solving algorithm configuration problems, practitioners typically start from some configuration (often, default or arbitrarily chosen settings) and then attempt to achieve improvements by modifying one parameter value at a time. If such an attempt does not result in improved performance, the modification is re- 
jected and the process continues from the previous configuration. This corresponds to an iterative first-improvement search in the space of configurations.

While the idea of performing local search in configuration space is appealing, considering the success achieved by similar methods on other hard combinatorial problems, iterative improvement is a very simplistic method that is limited to finding local optima. The key idea behind ParamILS is to combine more powerful stochastic local search (SLS) methods with mechanisms aimed at exploiting specific properties of algorithm configuration problems. The way in which this is done does not rely on an attempt to construct or utilise a model of good parameter configurations or of the impact of configuations on target algorithm perfomance; therefore, ParamILS is a model-free search procedure.

\subsection{The ParamILS Framework}

At the core of the ParamILS framework for automated algorithm configuration (Hutter et al, 2007b, 2009a) lies Iterated Local Search (ILS), a well-known and versatile stochastic local search method that has been applied with great success to a wide range of difficult combinatorial problems (see, e.g., Lourenço et al, 2002; Hoos and Stützle, 2004). ILS iteratively performs phases of simple local search, designed to rapidly reach or approach a locally optimal solution to the given problem instance, interspersed with so-called perturbation phases, whose purpose is to effectively escape from local optima. Starting from a local optimum $x$, in each iteration one perturbation phase is performed, followed by a local search phase, with the aim of reaching (or approaching) a new local optimum $x^{\prime}$. Then, a so-called acceptance criterion is used to decide whether to continue the search process from $x^{\prime}$ or whether to revert to the previous local optimum, $x$. Using this mechanism, ILS aims to solve a given problem instance by effectively exploring the space of its locally optimal solutions. At a lower level, ILS - like most SLS methods - visits (i.e., moves through) a series of candidate solutions such that at any given time, there is a current candidate solution, while keeping track of the incumbent (i.e., the best solution encountered so far).

ParamILS uses this generic SLS method to search for high-performance configurations of a given algorithm as follows (see also Figure 3). The search process is initialised by considering a given configuration (which would typically be the given target algorithm's default configuration) as well as $r$ further configurations that are chosen uniformly at random from the given configuration space. These $r+1$ configurations are evaluated in a way that is specific to the given ParamILS variant, and the best-performing configuration is selected as the starting point for the iterated local search process. This initialisation mechanism can be seen as a combination of the intuitive choice of starting from a user-defined configuration (such as the target algorithm's default settings) and a simple experimental design technique, where the latter makes it possible to exploit situations where the former represents a poor choice for the given set of benchmark instances. Clearly, there is a trade-off between 


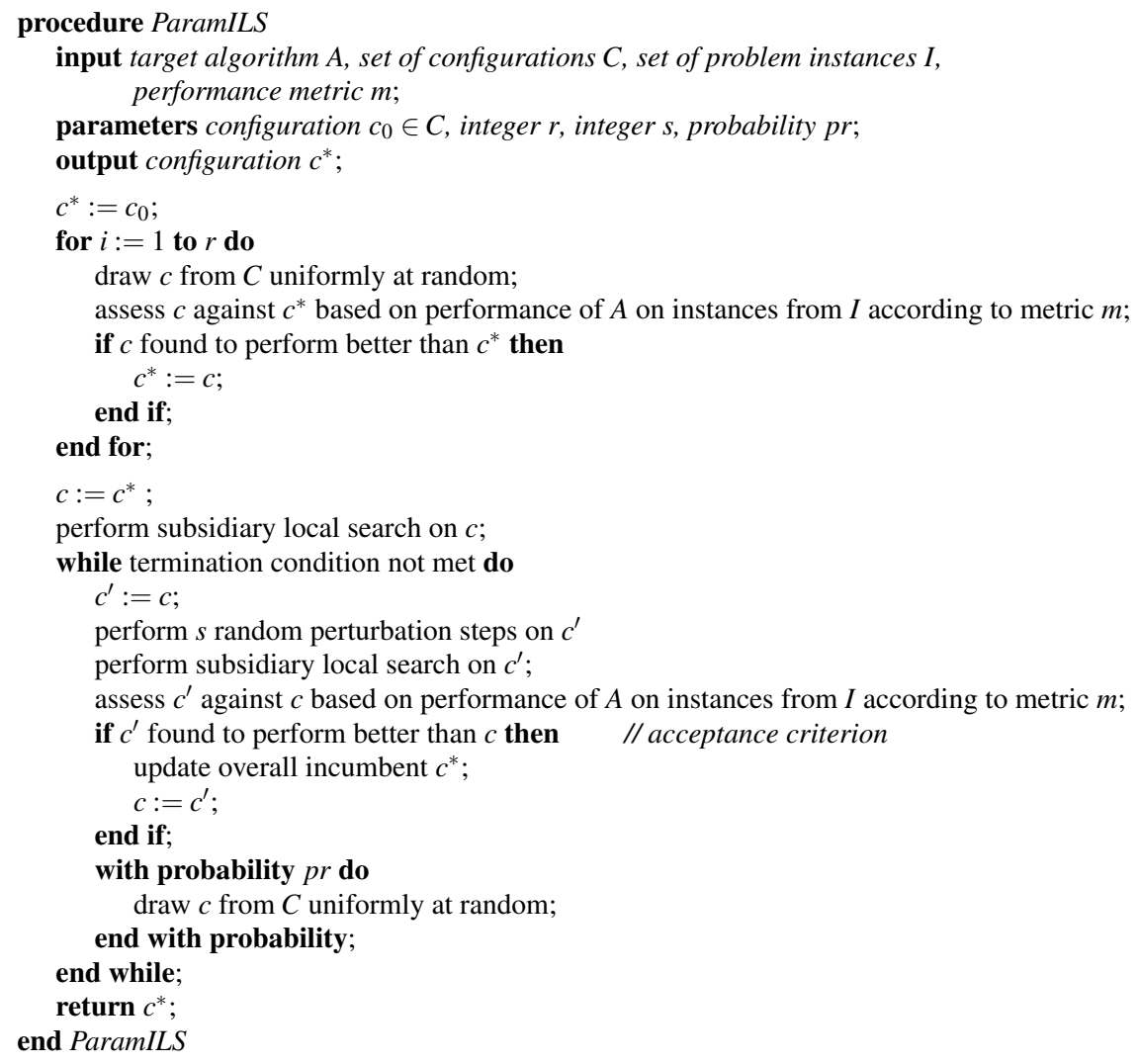

Fig. 3 High-level outline of ParamILS, as introduced by Hutter et al, 2007b; details are explained in the text.

the effort spent on evaluating randomly sampled configurations at this point and the effort used in the subsequent iterated local search process. Hutter et al (2009c) reported empirical results suggesting that $r=10$ results in better performance than $r=0$ and $r=100$ across a number of configuration scenarios. However, we suspect that more sophisticated initialisation procedures, in particular ones based on racing or sequential model-based optimisation techniques, might result in even better performance.

The subsidiary local search procedure used in ParamILS is based on the oneexchange neighbourhood induced by arbitrary changes in the values of a single target algorithm parameter. ParamILS supports conditional parameters by pruning neighbourhoods such that changes in inactive parameters are excluded from consideration; it also supports exclusion of (complete or partial) configurations explicitly declared 'forbidden' by the user. Using the one-exchange neighbourhood, ParamILS 
performs iterative first improvement search - an obvious choice, considering the computational cost of evaluating candidate configurations. We believe that larger neighbourhoods might prove effective in situations in which parameter effects are correlated, as well as in conjunction with mechanisms that recognise and exploit such dependencies in parameter response. Furthermore, search strategies other than iterative first improvement could be considered in variants of ParamILS that build and maintain reasonably accurate models of local parameter responses.

The perturbation procedure used in the ParamILS framework performs a fixed number, $s$, of steps chosen uniformly at random in the same one-exchange neighbourhood used during the local search phases. Computational experiments in which various fixed values of $s$ as well as several multiples of the number of target algorithm parameters were considered suggest that relatively small perturbations (i.e., $s=2$ ) are sufficient for obtaining good performance of the overall configuration procedure (Hutter et al, 2009c). Considering the use of iterative first improvement during the local search phases, this is not overly surprising; still, larger perturbations might be effective in combination with the future use of model-based techniques within the ParamILS framework.

While various acceptance criteria have been used in the literature on ILS, ParamILS uses one of the simplest mechanisms: Between two given candidate configurations, it always chooses the one with better observed performance; ties are broken in favour of the configuration reached in the most recent local search phase. This results in an overall behaviour of the iterated local search process equivalent to that of an iterative first improvement procedure searching the space of configurations reached by the subsidiary local search process. Considering once again the computational effort involved in each iteration of ParamILS, this is a natural choice; however, in cases where many iterations of ParamILS can be performed, and where the given configuration space contains attractive regions with many local minima, more complex acceptance criteria that provide additional search diversification (e.g., based on the Metropolis criterion) might prove useful.

In addition to the previously described perturbation procedure, ParamILS also uses a further diversification mechanism: At the end of each iteration, with a fixed probability $p r$ (by default set to 0.01 ), the current configuration is abandoned in favour of a new one that is chosen uniformly at random and serves as the starting point for the next iteration of the overall search process. This restart mechanism provides the basis for the probabilistic approximate completeness of FocusedILS, the more widely used of the two ParamILS variants discussed in the following. We believe that it also plays an important role towards achieving good performance in practice, although anecdotal empirical evidence suggests that additional diversification of the search process is required in order to eliminate occasionally occurring stagnation behaviour.

Finally, like the racing procedures discussed in the previous section, ParamILS performs blocking on problem instances, i.e., it ensures that comparisons between different configurations are always based on the same set of instances. This is important, since the intrinsic hardness of problem instances for any configuration of the given target algorithm may differ substantially. Furthermore, when used for op- 
timising the performance of a randomised target algorithm $A$, ParamILS also blocks on the pseudo-random number seeds used in each run of $A$; the main reason for this lies in the desire to avoid spurious performance differences in cases where the differences between two configurations have no impact on the behaviour of $A$.

\subsection{BasicILS}

The conceptually simplest way of assessing the performance of a configuration of a given target algorithm $A$ is to perform a fixed number of runs of $A$. This is precisely what happens in $\operatorname{BasicILS}(N)$, where the user-defined parameter $N$ specifies the number of target algorithm runs performed for each configuration to be assessed, using the same instances and pseudo-random number seeds. Applied to a randomised target algorithm $A$, BasicILS $(N)$ will only perform multiple runs per instance if $N$ exceeds the number of given problem instances; in this case, the list of runs performed is determined by a sequence of random permutations of the given set of instances, and the random number seed used in each run is determined uniformly at random.

This approach works well for configuration scenarios where a relatively small set of benchmark instances is representative for all instances of interest. Furthermore, the $N$ target algorithm runs per configuration can be performed independently in parallel. As for all ParamILS variants - and, indeed, for any SLS algorithm - further parallelisation can be achieved by performing multiple runs of BasicILS $(N)$ in parallel. Finally, in principle, it would be possible to perform multiple parallel runs of the subsidiary local search in each iteration or to evaluate multiple neighbours of a configuration in each search step independently in parallel.

\subsection{FocusedILS}

One drawback of BasicILS is that it tends to spend substantial effort in evaluating poor configurations, especially when used to configure a given target algorithm for minimised run-time. The only way to reduce that effort is to choose a small number of runs, $N$; however, this can (and often does) result in poor generalisation of performance to problem instances other than those used during the configuration process. FocusedILS addresses this problem by initially evaluating configurations using few target algorithm runs and subsequently performing additional runs to obtain increasingly precise performance estimates for promising configurations. We note that the idea of focussing the computational effort spent on evaluating configurations on candidates that have already shown promising performance is exactly the same as that underlying the concept of racing. However, unlike the previously discussed racing procedures, FocusedILS determines promising configurations heuristically rather than using statistical tests. 
The mechanism used by FocusedILS to assess configurations is based on the following concept of domination: Let $c_{1}$ and $c_{2}$ be configurations for which $N\left(c_{1}\right)$ and $N\left(c_{2}\right)$ target algorithm runs have been performed, respectively. As in the case of BasicILS, the runs performed for each configuration follow the same sequence of instances (and pseudo-random number seeds). Then $c_{1}$ dominates $c_{2}$ if, and only if, $N\left(c_{1}\right) \geq N\left(c_{2}\right)$ and the performance estimate for $c_{1}$ based on its first $N\left(c_{2}\right)$ runs is at least as good as that for $c_{2}$ based on all of its $N\left(c_{2}\right)$ runs. This definition incorporates the previously discussed idea of blocking, as configurations are compared based on their performance on a common set of instances (and pseudo-random number seeds).

Whenever FocusedILS decides that one configuration, $c_{1}$, performs better than another, $c_{2}$, it ensures that $c_{1}$ dominates $c_{2}$, by performing additional runs on either or both configurations. More precisely, when comparing two configurations, an additional run is first performed for the configuration whose performance estimates is based on fewer runs or, in the case of a tie, on both configurations. Then, as long as neither configuration dominates the other, further runs are performed based on the same criterion. Furthermore, when domination has been determined, FocusedILS performs additional runs for the winner of the comparison (ties are always broken in favour of more recently visited configurations). The number of these bonus runs is determined as the number of configurations visited since the last improving search step, i.e., since the last time a comparison between two configurations was decided in favour of the one that has been visited more recently. This mechanism ensures that the better a configuration appears to perform, the more thoroughly it is evaluated, especially in cases where a performance improvement is observed after a number of unsuccessful attempts.

As first established by Hutter et al (2007b), FocusedILS has an appealing theoretical property: With increasing run-time, the probability of finding a configuration with globally optimal performance on the given set of benchmark instances approaches one. This probabilistic approximate completeness (PAC) property follows from two key observations: Firstly, thanks to the previously mentioned probabilistic restart mechanism used in the ParamILS framework, over time, any configuration from a finite configuration space is visited arbitrarily often. Secondly, as the number of visits to a given configuration increases, so does the number of target algorithm runs FocusedILS performs on it, which causes the probability of mistakenly failing to recognise that its true performance on the given instance set is better than that of any other configuration it is compared against to approach zero. While this theoretical guarantee only concerns the behaviour of FocusedILS in the limit, as run-time approaches infinity, the mechanisms giving rise to it appear to be very effective in practice when dealing with surprisingly large configuration spaces (see, e.g., Hutter et al, 2007a, 2009a, 2010c). Nevertheless, stagnation of the search process has been observed in several cases and is typically ameliorated by performing multiple runs of FocusedILS independently in parallel, from which a single winning configuration is determined based on the performance observed on the set of benchmark instances used in those runs. We expect that by replacing the simplistic probabilistic restart mechanism, and possibly modifying the mechanism used for allocating the 
additional target algorithms runs to be performed when assessing configurations, stagnation can be prevented or overcome more effectively.

\subsection{Adaptive Capping}

Both, BasicILS and FocusedILS can be improved by limiting under certain conditions the time that is spent evaluating poorly performing configurations. The key idea is that when comparing two configurations $c_{1}$ and $c_{2}$, a situation may arise where, regardless of the results of any further runs, $c_{2}$ cannot match or exceed the performance of $c_{1}$ (Hutter et al, 2009a). This is illustrated by the following example, taken from Hutter et al (2009a): Consider a use of BasicILS(100) for minimising the expected run-time of a given target algorithm on a set of 100 benchmark instances, where configuration $c_{1}$ has solved all 100 instances in a total of $10 \mathrm{CPU}$ seconds, and $c_{2}$ has run for the same $10 \mathrm{CPU}$ seconds on the first instances without solving it. Clearly, we can safely terminate that latter run after $10+\varepsilon$ CPU seconds (for some small time $\varepsilon$ ), since the average run-time of $c_{2}$ must exceed $0.1 \mathrm{CPU}$ seconds, regardless of its performance in the remaining $N-1$ runs, and therefore be worse than that of $c_{1}$.

Based on this insight, the trajectory-preserving adaptive capping mechanism of Hutter et al (2009a) limits the effort spent on evaluating configurations based on comparing lower bounds on the performance for one configuration $c_{2}$ against upper bounds (or exact values) on that of another configuration $c_{1}$ based on the results of given sets of runs for $c_{1}$ and $c_{2}$. We note that this corresponds to a notion of racing, where each of the two configurations is working independently through a given number of runs, but the race is terminated as soon as the winner can be determined with certainty. Apart from the potential for savings in running time, the use of trajectory-preserving capping does not change the behaviour of ParamILS.

A heuristic generalisation of this capping mechanism makes it possible to achieve even greater speedups, albeit at the price of possibly substantial changes to the search trajectory followed by the configuration procedure. The key idea behind this generalisation (dubbed aggressive capping) is to additionally bound the time allowed for evaluating configurations based on the performance observed for the current incumbent, i.e., the best-performing configuration encountered since the beginning of the ParamILS run. The additional bound is obtained by multiplying the performance estimate of the incumbent by a constant $b m$ called the bound multiplier. Formally, for $b m=\infty$, the additional bound becomes inactive (assuming the performance measure is to be minimised), and the behaviour of trajectory-preserving capping is obtained. For $b m=1$, on the other hand, a very aggressive heuristic is obtained, which limits the evaluation of any configuration to the time spent on evaluating the current incumbent. In practice, $b m=2$ appears to result in good performance and is used as a default setting in ParamILS. Despite its heuristic nature, this modified capping mechanism preserves the PAC property of FocusedILS. 
Although Hutter et al (2009a) spelled out their adaptive capping mechanisms for the performance objective of minimising a target algorithm's mean run-time only, these mechanisms generalise to other objectives in a rather straightforward way (a discussion of capping in the context of minimising quantiles of run-time is found in Ch. 7 of the dissertation of Hutter, 2009). We note, however, that - especially when several target algorithm runs are conducted in parallel - adaptive capping would be most effective in the case of run-time minimisation. Particularly substantial savings can be achieved during the assessment of the $r+1$ configurations considered during initialisation of the search process, as well as towards the end of each local search phase. Finally, it should be noted that adaptive capping mechanisms can be used in the context of configuration procedures other than ParamILS; for example, Hutter et al (2009a) mention substantial speedups achieved by using adaptive capping in combination with simple random sampling (the same procedure as used during the initialisation of ParamILS).

\subsection{Applications}

ParamILS variants, and in particular FocusedILS, have been very successfully applied to a broad range of high-performance algorithms for several hard combinatorial problems. An early version of FocusedILS was used by Thachuk et al (2007) to configure a replica-exchange Monte Carlo (REMC) search procedure for the 2D and 3D HP protein structure prediction problems; the performance objective was to minimise mean run-time for finding ground states for a given set of sequences in these abstract, but prominent models of protein structure, and the resulting configurations of the REMC procedure represented a considerable improvement in the state of the art in solving these challenging problems.

FocusedILS has also been used in a series of studies leading to considerable advances in the state of the art of solving the satisfiability problem in propositional logic, one of the most widely studied $\mathscr{N} \mathscr{P}$-hard problems in computing science. Hutter et al (2007a) applied this procedure to SPEAR, a complete, DPLL-type SAT solver with 26 parameters (10 of which are categorical), which jointly give rise to a total of $8.34 \cdot 10^{17}$ possible configurations. The design of SPEAR was influenced considerably by the availability of a powerful configuration tool such as FocusedILS, whose application ultimately produced configurations that solved a given set of SAT-encoded software verification problems about 100 times faster than previous state-of-the-art solvers for these types of SAT instances and won the first prize in the QF_BV category of the 2007 Satisfiability Modulo Theories (SMT) Competition.

KhudaBukhsh et al (2009) used FocusedILS to find performance-optimised instantiations of SATenstein-LS, a highly parametric framework for stochastic local search (SLS) algorithms for SAT. This framework was derived from components found in a broad range of high-performance SLS-based SAT solvers; its 41 parameters induce a configuration space of size $4.82 \cdot 10^{12}$. Using FocusedILS, performance 
improvements of up to three orders of magnitudes were achieved over the previous best-performing SLS algorithms for various types of SAT instances, for several of which SLS-based solvers are the most effective SAT algorithms overall. Several automatically determined configurations of SATenstein-LS were used in the most recent SATzilla solvers, which led the field in the 2009 SAT Competition, winning prizes in 5 of the 9 main categories (Xu et al, 2009).

Very recently, Xu et al (2010) used FocusedILS in an iterative fashion to obtain sets of configurations of SATenstein-LS that were then used in combination with state-of-the-art per-instance algorithm selection techniques (here: SATzilla). In each iteration of the overall procedure, dubbed Hydra, FocusedILS was used to find configurations that would best complement a given portfolio-based per-instance algorithm selector. This approach resulted in a portfolio-based SAT solver that, while derived in a fully automated fashion from a single, highly parameterised algorithm, reached state-of-the-art performance across a wide range of benchmark instances.

Tompkins and Hoos (2010) applied FocusedILS to a new, flexible framework for SLS-based SAT solvers called VE-Sampler (which is conceptually orthogonal to the previously mentioned SATenstein-LS framework). VE-Sampler has a large number of categorical and conditional parameters, which jointly give rise to more than $10^{50}$ distinct configurations, and using FocusedILS, configurations could be found that were shown to solve two well-known sets of SAT-encoded software verification problems between 3.6 and 9 times faster than previous state-of-the-art SLS-based SAT solvers for these types of SAT instances.

Chiarandini et al (2008) used FocusedILS to configure a hybrid, modular SLS algorithm for a challenging university timetabling problem that subsequently placed third in Track 2 of the Second International Timetabling Competition (ITC 2007). The configuration space considered in this context was relatively small (7 parameters, 50400 configurations), but the use of automated algorithm configuration made it possible to achieve close to state-of-the-art performance within very limited human development time and without relying on deep and extensive domain expertise. In subsequent work, Fawcett et al (2009) expanded the design space by parameterising additional parts of the solver, and - using multiple rounds of FocusedILS with different performance objectives - obtained a configuration that was demonstrated to substantially improve upon the state of the art for solving the post-enrolment course timetabling problem considered in Track 2 of ITC 2007. The overall performance metric used in these studies (and in the competition) was solution quality achieved by the target solver after a fixed amount of time.

Recently, Hutter et al (2010c) reported substantial improvements in the performance of several prominent solvers for mixed integer programming (MIP) problems, including the widely used industrial CPLEX solver. In the case of CPLEX, FocusedILS was used to configure 76 parameters, most of which are categorical (and some conditional), giving rise to a configuration space of size $1.9 \cdot 10^{47}$. Despite the fact that the default parameter settings for CPLEX are known to have been chosen carefully, based on a considerable amount of thorough experimentation, substantial performance improvements were obtained for many prominent types of MIP instances, both, in terms of time required for finding optimal solutions (and proving 
optimality) and of minimising the solution quality (optimality gap) achieved within a fixed amount of time (speedup factors beween 1.98 and 52.3, and gap reduction factors between 1.26 and 8.65). Similarly impressive results were achieved for another commercial MIP solver, Gurobi, and a prominent open-source MIP solver, lpsolve.

Finally, Hutter et al (2009c) reported a successful meta-configuration experiment, in which BasicILS was used to optimise the four parameters that control the behaviour of FocusedILS. BasicILS was chosen as the meta-configurator, since its runs can be parallelised in a straightforward manner. At least partly because of the enormous computational cost associated with this experiment (where each target algorithm run corresponds to solving an algorithm configuration task, and hence to executing many costly runs of the algorithm configured in that task, which itself solved instances of a $\mathscr{N P}$-hard problem, such as SAT), only marginal improvements in the performance of the configurator, FocusedILS, on a number of previously studied configuration benchmarks could be achieved.

The target algorithms considered in most of these applications have continuous parameters, and up to this point, ParamILS requires these parameters to be discretised. While in principle, finding reasonable discretisations (i.e., ones whose use does not cause major losses in the performance of the configurations found by ParamILS) could be difficult, in most cases, generic approaches, such as even or geometric subdivisions of a given interval, seem to give good results. Where this is not the case, multiple runs of the configuration procedure can be used to iteratively refine the domains of continuous parameters. The same approach can be used to extend domains in cases where parameter values in an optimised configuration lie at the boundary of their respective domains. Nevertheless, the development of ParamILS variants that natively deal with continuous parameters and support dynamic extensions of parameter domains remains an interesting direction for future work.

\section{Sequential Model-Based Optimisation}

A potential disadvantage of the model-free search approach underlying ParamILS is that it makes no explicit attempt to recognise and benefit from regularities in a given configuration space. The model-based search paradigm underlying the approach discussed in this section, on the other hand, uses the information gained from the configurations evaluated so far to build (and maintain) a model of the configuration space, based on which configurations are chosen to be evaluated in the future. We note that, as also pointed out by its authors, the Iterative F-Race procedure (I/FRace) discussed in Section 2.2 of this chapter, is a model-based configuration procedure in this sense. But unlike I/F-Race, the models used by the methods discussed in the following capture directly the dependency of target algorithm performance on parameter settings. These response surface models can be used as surrogates for the actual parameter response of a given target algorithm and provide the basis 


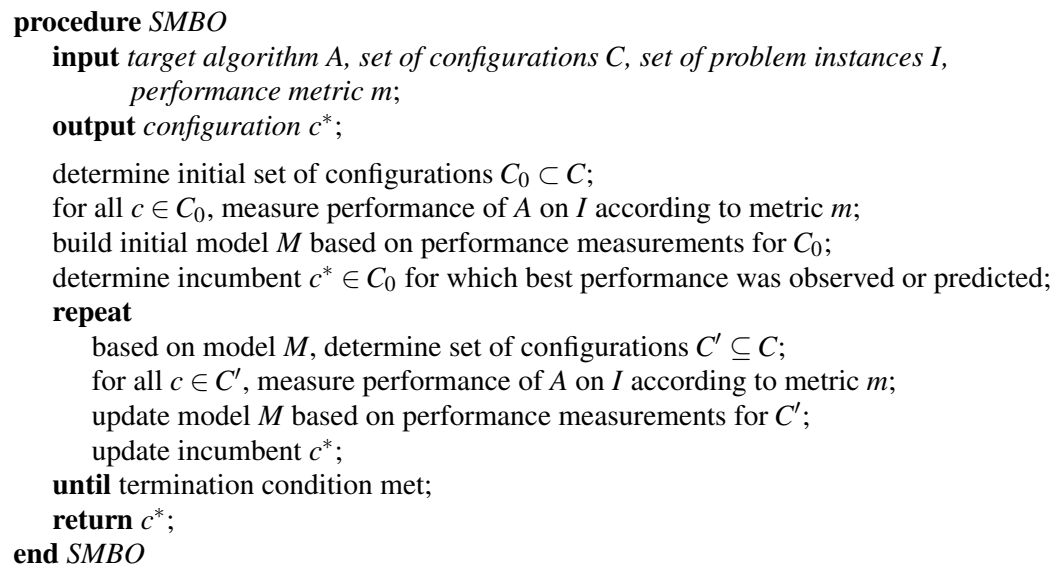

Fig. 4 High-level outline of the general sequential model-based optimisation approach to automated algorithm configuration; model $M$ is used to predict the performance of configurations that have not (yet) been evaluated, and set $C^{\prime}$ is typically chosen to contain configurations expected to perform well based on those predictions. Details of various algorithms following this approach are explained in the text.

for determining promising configurations at any stage of an iterative model-based search procedure; this generic approach is known as sequential model-based optimisation $(S M B O)$ and can be seen as a special case of sequential analysis - a broader area within statistics that also comprises sequential hypothesis testing and so-called multi-armed bandits. An outline of SMBO for algorithm configuration is shown in Figure 4; in principle, performance measurements for multiple configurations can be performed independently in parallel.

The setting considered in almost all work on sequential model-based optimisation procedures up to this day is known as the black-box optimisation problem: Given an unknown function $f$ and a space of possible inputs $X$, find an input $x \in X$ that optimises $f$ based on measurements of $f$ on a series of inputs. The function to be optimised, $f$, may be deterministic or stochastic; in the latter case, measurements are subject to random noise and formally, the values of $f$ are random variables. Algorithm configuration can be seen as a special case of black-box optimisation, where the function to be optimised is the performance $m$ of an algorithm $A$ on a set of problem instances $I$. However, in contrast to algorithm configuration procedures such as FocusedILS or F-Race, black-box optimisation procedures do not take into account the fact that approximate measurements can be obtained at lower computational cost, by running $A$ on subsets of $I$, and that by blocking on instances (and pseudorandom number seeds), performance measurements for different configurations can be compared more meaningfully; furthermore, they have no means of exploiting knowledge about the instances in $I$ acquired from earlier target algorithm runs. 
Because black-box function optimisation is somewhat more general than algorithm configuration, and methods for solving black-box function are easily applicable to modelling and optimising the response of a wide range of systems, in the following we use standard terminology from the statistics literature on experimental design, in particular, design point for elements of the given input space $X$ and response for values of the unknown function $f$. In the context of algorithm configuration, design points correspond to configurations of a given target algorithm $A$, and response values represent $A$ 's performance $m$ on instance set $I$. A unified, more technical presentation of the methods covered in this section can be found in the dissertation of Hutter (2009), and further details are provided in the original articles referenced throughout.

\subsection{The EGO Algorithm}

The efficient global optimisation (EGO) algorithm for black-box function optimisation by Jones et al (1998) uses a response surface model obtained via noise-free Gaussian process regression in combination with an expected improvement criterion for selecting the next configuration to be evaluated. The noise-free Gaussian process (GP) model utilised by EGO is also known as the DACE model, after its prominent use in earlier work by Sacks et al (1989). It defines for every input $x$ a random variable $\hat{F}(x)$ that characterises the uncertainty over the true response value $f(x)$ at point $x$.

The model-based optimisation process carried out by EGO starts with about $10 \cdot k$ design points determined using a $k$-dimensional space-filling Latin hypercube design (LHD). After measuring the response values for these values, the $2 \cdot k+2$ parameters of a DACE model are fit to the pairs of design points and response values, using maximum likelihood estimates (as described by Jones et al, 1998, this can be partially done in closed form). The resulting model is assessed by means of socalled standardized cross-validated residuals, which reflect the degree to which predictions made by the model agree with the observed response values on the design points used for constructing the model. If the model is deemed unsatisfactory, the response values may be transformed using a log-or inverse-transform (i.e., modified by applying the function $\ln y$ or $1 / y$ ) and the model fitted again.

After a satisfactory initial model has been obtained, it is used in conjunction with an expected improvement criterion to determine a new design point to be evaluated. The expected improvement measure used in this context uses the current DACE model $M$ to estimate the expected improvement over the best response value measured so far, $f_{\min }$, at any given design point $x$, and is formally defined as $E I(x):=E\left[\max \left\{f_{\min }-\hat{F}(x), 0\right\}\right]$, where $\hat{F}(x)$ is the random variable describing the response for a design point $x$ according to model $M$. Using a closed-form expression for this measure given by Jones et al (1998) and a branch \& bound search method (which can be enhanced heuristically), the EGO algorithm then determines a design point $x^{\prime}$ with maximal expected improvement $E I\left(x^{\prime}\right)$. If $E I\left(x^{\prime}\right)$ is less than $1 \%$ of the 
current incumbent, the procedure terminates. Otherwise, the response value $f\left(x^{\prime}\right)$ is measured, and the DACE model is refitted on the previous set of data extended by the pair $\left(x^{\prime}, f\left(x^{\prime}\right)\right)$, and a new iteration begins, in which the updated model is used to determine the next design point using the same process that yielded $x^{\prime}$.

Note that in every iteration of this process, the DACE model has to be fitted, which involves a matrix inversion of $\operatorname{cost} O\left(n^{3}\right)$, where $n$ is the number of design points used. Depending on the cost of measuring the response value for a given design point, this may represent a substantial computational overhead. Furthermore, the noise-free Gaussian process model used in EGO cannot directly characterise the stochastic responses obtained when solving algorithm configuration problems involving randomised target algorithms.

\subsection{Sequential Kriging Optimisation and Sequential Parameter Optimisation}

We now discuss two black-box optimisation procedures that deal with stochastic responses, as encountered when modelling phenomena subject to observation noise or configuring randomised algorithms.

The first of these, known as Sequential Kriging Optimisation (Huang et al, 2006) estimates a Gaussian process (GP) model (also known as a kriging model) directly from samples (i.e., noisy measurements) of response values. Similar to EGO, SKO starts with an LHD of $10 \cdot k$ design points, for which response values are sampled. To facilitate initial estimates of the observation noise, one additional sample is then drawn for each of the $k$ best of these design points, after which a Gaussian process model $M$ is fitted directly to the resulting set of $11 \cdot k$ pairs of design points and corresponding response values. The resulting model $M$ is then assessed and possibly modified based on a transformation of the response, as in the EGO algorithm.

Next, an incumbent design point is determined based on model $M$, by minimising the expression $\mu(x)+\sigma(x)$ using the Nelder-Mead Simplex algorithm (Nelder and Mead, 1965), where $\mu(x)$ and $\sigma(x)$ are the mean and standard deviation predicted by $M$ for input $x$, and the minimisation is over the design points used in constructing the model. This risk-adverse strategy is less easily misled by inaccurate estimates of the mean response value than a minimisation of the predicted mean only. The next design point to be evaluated is determined based on model $M$ using an augmented expected improvement measure, designed to steer the process away from design points with low predictive variance. This augmented expected improvement measure is formally defined as

$$
E I^{\prime}(x):=E\left[\max \left\{\hat{f}_{\min }-\hat{F}(x), 0\right\}\right] \cdot\left(1-\sigma_{\varepsilon} / \sqrt{s^{2}(x)-\sigma_{\varepsilon}^{2}}\right),
$$

where $\hat{f}_{\text {min }}$ is the model's prediction for the current best input (as in EGO, obtained by considering all design points used for building the model), $\hat{F}(x)$ is the random 
variable describing the response for a design point $x$ according to model $M, \sigma_{\varepsilon}$ is the standard deviation of the measurement noise (assumed to be identical for all inputs), and $s^{2}(x)$ is the variance of the response $\hat{F}(x)$ given by the model at point $x$, where the second term in the product decreases as the predictions of $M$ become more accurate. Based on the given model $M$, the next design point to be evaluated, $x^{\prime}$, is determined by maximising $E I^{\prime}(x)$ using the Nelder-Mead Simplex algorithm (Nelder and Mead, 1965). Next, the model is refitted, taking into account $x^{\prime}$ and a response value sampled at $x^{\prime}$, and a new iteration begins, in which the updated model is used to determine the next design point using the same process that yielded $x^{\prime}$. If the maximum $E I^{\prime}(x)$ values from $d+1$ successive iterations all fall below a userdefined threshold, the iterative sampling process is terminated. (This treshold can be specified as an absolute value or as a fraction of the difference between the largest and smallest observed response values.)

Unfortunately, SKO assumes that the variability of the response values at each design point is characterised by a Gaussian distribution, and that the standard deviations of those distributions are the same across the entire design space. Both of these assumptions are problematic in the context of configuring randomised algorithms, particularly when minimising run-time (see, e.g., Hoos and Stützle, 2004). Furthermore, the time required for fitting the model in each iteration of SKO is cubic in the number of response values sampled, which can represent a substantial computational burden.

The Sequential Parameter Optimisation (SPO) procedure by Bartz-Beielstein et al (2005) follows a fundamentally different strategy to deal with noisy response measurements: ${ }^{3}$ Rather than fitting a Gaussian process model directly to the a set of sampled responses, for each design point $x$, the measure to be optimised is estimated empirically based on all samples taken at $x$, and a noise-free Gaussian process model (like the one used in the EGO algorithm) is fitted to the resulting data. In contrast to the approach taken by SKO, this makes it possible to optimise arbitrary statistics of the noisy function values, i.e., in the case of algorithm configuration, of the given target algorithm's run-time distributions; examples of such statistics are the mean, median, arbitrary quantiles, but also measures of variability, as well as combinations of measures of location and variability. Another advantage of this approach is its substantially lower computational complexity: While SKO requires time cubic in the number of function values sampled, SPO's run-time is only cubic in the number of distinct design points - typically a much lower number.

Like SKO and EGO, SPO uses a Latin hypercube design as a basis for constructing the initial model; however, SPO chooses $d$ design points and samples $r$ response values for each of these, where $d$ and $r$ are specified by the user (the default value of $r$ is 2). Based on these samples, empirical estimates of the measure to be optimised

\footnotetext{
${ }^{3}$ In the literature, the term sequential parameter optimisation is also used to refer to a broad methodological framework encompassing fully automated as well as interactive approaches for understanding and optimising an algorithm's performance in response to its parameter settings. Here, as in the work of Hutter et al (2009b), we use the term more narrowly to refer to the fully automated SMBO procedures implemented in various versions of the Sequential Parameter Optimization Toolbox (SPOT) by Bartz-Beielstein et al, 2008.
} 
are calculated for each design point, and the point with the best resulting value is chosen as the initial incumbent. Next, a noise-free Gaussian process model is fitted to the resulting set of $d$ pairs of design points and empirical response statistics. This model, $M$, is sampled for 10000 design points chosen uniformly at random, ${ }^{4}$ and the best $j$ of these according to an expected improvement (EI) measure are selected for further evaluation, where $j$ is a user-defined number with a default setting of 1 . The EI measure used in this context is formally defined as

$$
E I^{2}(x):=E\left[\left(f_{\min }-\hat{F}(x)\right)^{2}\right]=E^{2}\left[f_{\min }-\hat{F}(x)\right]+\operatorname{Var}\left[f_{\min }-\hat{F}(x)\right],
$$

where $f_{\min }$ is the best value of the measure to be optimised observed so far, and $\hat{F}(x)$ is the distribution over the predictions obtained from model $M$ at design point $x$. This EI measure has been introduced by Schonlau et al (1998) with the aim of encouraging the exploration of design points for which the current model produces highly uncertain predictions.

At each of the design points determined in this way, $r$ new response values are measured. Furthermore, additional response values are measured for the current incumbent to ensure that it is evaluated based on as many samples as available for any of the new design points. Then, the best of all the design points considered so far, according to the given measure to be optimised, is selected as the new incumbent (with ties broken uniformly at random). If the design point thus selected has been an incumbent at any point earlier in the search process, $r$ is increased; in SPO version 0.3 (Bartz-Beielstein et al, 2005), $r$ is doubled, while in the newer version 0.4 (Bartz-Beielstein, 2006), it is merely incremented by one, and in both cases, values of $r$ are limited to a user-specified maximum value $r_{\max }$. At this point, a new iteration of SPO begins, in which a noise-free GP is fitted on the augmented set of data.

\subsection{Recent Variants of Sequential Parameter Optimisation: $\mathrm{SPO}^{+}$and TB-SPO}

Based on a detailed investigation of the core components of the SPO algorithm, Hutter et al (2009b) introduced a variant called $\mathrm{SPO}^{+}$that shows considerably more robust performance on standard benchmarks than the SPO 0.3 and SPO 0.4 algorithms described previously.

The main difference between $\mathrm{SPO}^{+}$and the previous SPO procedures lies in the way in which new design points are accepted as incumbents. Inspired by FocusedILS, $\mathrm{SPO}^{+}$uses a mechanism that never chooses a new incumbent $\hat{x}^{\prime}$ without ensuring that at least as many responses have been sampled at $\hat{x}^{\prime}$ as at any other design point $x \neq \hat{x}^{\prime}$. To achieve this, for any challenger to the current incumbent $\hat{x}$, i.e., for any design point $x^{\prime}$ that appears to represent an improvement over $\hat{x}$ based

${ }^{4}$ We note that the use of a space-filling design, such as an LHD, should in principle yield better results, if implemented sufficiently efficiently. 
on the samples taken so far, additional response values are sampled until either $x^{\prime}$ ceases to represent an improvement, or the number of response values sampled at $x^{\prime}$ reaches that taken at $\hat{x}$, with $x^{\prime}$ still winning the comparison with $\hat{x}$ based on the respective samples; only in the latter case, $x^{\prime}$ becomes the new incumbent, while in the former case it is dismissed, and as many additional response values are sampled for $\hat{x}$ as newly measured for $x^{\prime}$.

The new response values determined for a challenger $x^{\prime}$ are sampled in batches, with the number of new samples taken doubling in each successive batch. As noted by Hutter et al (2009b), using this mechanism, rejection of challengers is done in a rather aggressive, heuristic manner, and frequently occurs after only a single response value has been sampled at $x^{\prime}-$ long before a statistical test could conclude that the $x^{\prime}$ is worse than the current incumbent.

The Time-bounded Sequential Parameter Optimisation (TB-SPO) algorithm by Hutter et al (2010b) introduces a number of further modifications to the SMBO framework underlying the previously described SPO variants. In particular, in contrast to all SMBO procedures discussed so far, TB-SPO does not construct its initial model based on a large sets of samples determined using a Latin hypercube design, but rather interleaves response measurements at randomly chosen points with ones taken at points that appear to be promising based on the current model. The initial model is based on a single sample only; when used for algorithm configuration, where the black-box function to be optimised represents the output of a parameterised algorithm, the default configuration for the algorithm would be used as the design point at which this initial sample is taken. At any stage of the iterative modelbased search process that follows, response values are sampled at a series of design points in which odd-numbered points are determined by optimising an expected improvement measure (as is done in $\mathrm{SPO}^{+}$), while even-numbered points are sampled uniformly at random from the given design space. (Mechanisms that achieve a different balance between promising and randomly chosen design points could lead to better performance but have not been explored so far.)

The number of design points at which response values are sampled between any two updates to the model is determined based on the time $t$ required for constructing a new model and the search for promising parameter settings; to be precise, after at least two design points have been evaluated, further points are considered until the time used for evaluating design points since the last model update exceeds a userdefined multiple (or fraction) of the overhead $t$.

Finally, in order to reduce the computational overhead incurred by the model construction process, TB-SPO uses an approximate version of the standard Gaussian process models found in the other SPO variants. This so-called projected process $(P P)$ approximation is based on the idea to only explicitly represent a randomly sampled subset of the given data points (here: pairs of input and response values) when building the Gaussian process model; if this subset comprises $s$ data points, while the complete set has $n$ data points, the time complexity of fitting a GP model decreases from $O\left(n^{3}\right)$ to $O\left((s+n) \cdot s^{2}\right)$, while the time required for predicting a response value (mean and variance of the predictive distribution at a given design point) decreases from $O\left(n^{2}\right)$ to $O\left(s^{2}\right)$ (Rasmussen and Williams, 2006). In the con- 
text of an SMBO procedure, this will typically lead to substantial savings, since the number of data points available increases over time, and $n$ can easily reach values of several thousand, while effective PP approximations can be based on constant-size subsets with $s$ no larger than 300 (Hutter et al, 2010b). (Details on other, minor differences between TB-SPO and $\mathrm{SPO}^{+}$can be found in the original article by Hutter et al, 2010b.)

\subsection{Applications}

As mentioned earlier, sequential model-based optimisation methods have primarily been developed for the optimisation of black-box functions, but can obviously be applied to algorithm configuration problems by defining the function to be optimised to be the performance of a target observed algorithm applied to one or more benchmark instances. The design points are thus algorithm configurations, and the response values capture the performance of $A$ on the given benchmark instance(s) according to some performance measure $m$. In principle, SMBO procedures like those described earlier in this section can be applied to optimise a target algorithm on a set $I$ of benchmark instances by using a measure $m$ that captures the performance on the entire set $I$; however, as discussed earlier for the case of BasicILS, this tends to quickly become impractical as the size of $I$ grows. Therefore, the empirical evaluation of SMBO procedures tends to be focussed on performance optimisation on single benchmark instances. Furthermore, because of the nature of the response surface models used, SMBO methods are usually restricted to dealing with realand integer-valued target algorithm parameters (although very recently, Hutter et al, 2011, have introduced techniques that can handle categorical parameters).

Following an example from Bartz-Beielstein et al (2008), the SPO variants discussed in this section have been empirically evaluated using CMA-ES (Hansen, 2006; Hansen and Kern, 2004; Hansen and Ostermeier, 2001) - one of the bestperforming gradient-free numerical optimisation procedures currently known - on several standard benchmark functions from the literature on gradient-free numerical optimisation (see, e.g., Hansen and Kern, 2004). The configuration space considered in these examples, which involve the convex Sphere function as well as the nonconvex Ackley, Griewank and Rastrigin functions, is spanned by three real- and one integer-valued parameters of CMA-ES, and the performance measure was solution quality achieved after a fixed number of evaluations of the respective benchmark function. The empirical results reported by Hutter et al (2009b) for CMA-ES applied to the 10-dimensional instances of these functions indicate that $\mathrm{SPO}^{+}$tends to perform significantly better than SPO 0.3 and 0.4 , which in turn appear to perform substantially better than SKO. In addition, Hutter et al (2009b) considered the minimisation of median number of search steps required by SAPS (Hutter et al, 2002), a well-known stochastic local search algorithm for SAT, to solve a single benchmark instance obtained from encoding a widely studied quasi-group completion problem into SAT; in this case, four continuous parameters were optimised. The results from 
that experiment confirmed that $\mathrm{SPO}^{+}$tends to perform better than previous SPO variants and suggest that, at least on some configuration problems with a relatively modest number of predominently real-valued parameters, it can also yield slightly better results than FocusedILS when allowed the same number of target algorithm runs.

TB-SPO has been empirically compared against $\mathrm{SPO}^{+}$and FocusedILS on relatively simple algorithm configuration tasks involving the well-known SAT solver SAPS (Hutter et al, 2002), with 4 continuous parameters, running on single SAT instances. In these experiments, TB-SPO was shown to perform significantly better than $\mathrm{SPO}^{+}$(sometimes achieving over 250 -fold speedups), and moderately better than FocusedILS (Hutter et al, 2010b). However, it is important to keep in mind that, unlike TB-SPO (and all other SMBO procedures covered in this section), FocusedILS explicitly deals with multiple problem instances, and can therefore be expected to perform substantially better on realistic algorithm configuration tasks. Furthermore, while SMBO procedures like TB-SPO do not require continuous algorithm parameters to be discretised, they presently cannot deal with conditional parameters, which are routinely encountered in the more challenging algorithm configuration tasks on which FocusedILS has been shown to be quite effective.

\section{Other Approaches}

In addition to the methods covered in the previous sections, there are many other procedures described in the literature that can, at least in principle, be applied to the algorithm configuration problem considered here.

Experimental design methods, such as full or fractional factorial designs, stratified random sampling, Latin hypercube designs and various other types of spacefilling and uniform designs (see, e.g., Santner et al, 2003) are applicable to algorithm configuration, but per se do not take into account one fundamental aspect of the problem: Namely, that we are interested in performance on multiple instances and have control over the number of instances used for evaluating any given configuration. Furthermore, when minimizing the run-time of the given target algorithm (a very common performance objective in algorithm configuration and parameter tuning), it is typically necessary to work with censored data from incomplete runs, and it can be beneficial to cut off runs early (as done by the adaptive capping strategy explained in Section 3.4). Perhaps more importantly, they lack the heuristic guidance that is often crucial for searching large configuration spaces effectively.

Nevertheless, these simple design methods are sometimes used for the initialisation of more complex procedures (see Section 4 and the work by Adenso-Diaz and Laguna, 2006, which will be discussed in slightly more detail later). There is also some evidence that in certain cases, a method as simple as uniform random sampling, when augmented with adaptive capping or with the mechanism used by TB-SPO for evaluating configurations, can be quite effective (see recent work by Hutter et al, 2010b). 
In principle, gradient-free numerical optimisation methods are directly applicable to parameter tuning problems, provided that all parameters are real-valued (and that there are no parameter dependencies, such as conditional parameters). Prominent and relatively recent methods that appear to be particularly suitable in this context are the covariance matrix adaptation evolution strategy (CMA-ES) by Hansen and Ostermeier (2001) and the mesh adaptive direct search (MADS) algorithms by Audet and Orban (2006). Similarly, it is possible to use gradient-based numerical optimisation procedures - in particular, quasi-Newton methods such as the BroydenFletcher-Goldfarb-Shanno (BFGS) algorithm (see, e.g., Nocedal and Wright, 2006) - in conjunction with suitable methods for estimating or approximating gradient information. However, in order to be applied in the context of configuring target algorithms with categorical and conditional parameters, these methods would require non-obvious modifications; we also expect that in order to be reasonably effective, they would need to be augmented with mechanisms for dealing with multiple problem instances and capped (or censored) runs. The same holds for standard methods for solving stochastic optimisation problems (see, e.g., Spall, 2003).

The CALIBRA algorithm by Adenso-Diaz and Laguna (2006), on the other hand, has been specifically designed for parameter tuning tasks. It uses a specific types of fractional factorial designs from the well-known Taguchi methodology in combination with multiple runs of a local search procedure that gradually refines the region of interest. Unfortunately, CALIBRA can handle no more than five parameters, all of which need to be ordinal (the limitation to five parameters stems from the specific fractional designs used at the core of the procedure).

The CLASS system by Fukunaga $(2002,2004)$ is based on a genetic programming approach; it has been specifically built for searching a potentially unbounded space of the heuristic variable selection method used in a SLS-based SAT solver. Like most genetic programming approaches, CLASS closely links the specification of the configuration space and the evolutionary algorithm used for exploring this space.

The Composer system developed by Gratch and Dejong (1992) is based on an iterative improvement procedure not unlike that used in ParamILS; this procedure is conceptually related to racing techniques in that it moves to a new configuration only after gathering sufficient statistical evidence to conclude that this new configuration performs significantly better than the current one. In a prominent application, Gratch and Chien (1996) used the Composer system to optimise five parameters of an algorithm for scheduling communication between a spacecraft and a set of ground-based antennas.

Ansótegui et al (2009) recently developed a gender-based genetic algorithm for solving algorithm configuration problems. Their GGA procedure supports categorical, ordinal and real-valued parameters; it also allows its user to express independencies between parameter effects by means of so-called variable trees - a concept that could be of particular interest in the context of algorithm configuration problems where such independencies are known by construction, or heuristic methods are are available for detecting (approximate) independencies. Although there is some evidence that GGA can solve some moderately difficult configuration prob- 
lems more effectively than FocusedILS without capping (Ansótegui et al, 2009), it appears to be unable to reach the performance of FocusedILS version 2.3 with aggressive capping on the most challenging configurations problems (Hutter et al, 2010a). Unfortunately, GGA also offers less flexibility than FocusedILS in terms of the performance metric to be optimised. Further algorithm configuration procedures based on evolutionary algorithms are covered in Chapter ?? of this book.

Finally, work originating from the Ph.D. project of Hutter (2009) has recently overcome two major limitations of the sequential model-based optimisation methods discussed in Section 4 of this chapter by introducing a procedure that can handle categorical parameters while explicitly exploiting the fact that performance is evaluated on a set of problem instances. There is some evidence that this procedure, dubbed Sequential Model-based Algorithm Configuration (SMAC), can, at least on some challenging configuration benchmarks, reach and sometimes exceed the performance of FocusedILS (Hutter et al, 2011), and we are convinced that at least in cases where the parameter response of a given target algorithm is reasonably regular and performance evaluations are very costly, such advanced SMBO methods hold great promise.

\section{Conclusions and Future Work}

Automated algorithm configuration and parameter tuning methods have been developed and used for more than a decade, and many of the fundamental techniques date back even further. However, it has only recently become possible to effectively solve complex configuration problems involving target algorithms with dozens of parameters, which are often categorical and conditional. This success is based in part on the increased availability of computational resources, but has mostly been enabled by methodological advances underlying recent configuration procedures.

Still, we see much room (and, indeed, need) for future work on automated algorithm configuration and parameter tuning methods. We believe that in developing such methods, the fundamental features underlying all three types of methods discussed in this chapter can all play an important role, and that the best methods will employ combinations of these. We further believe that different configuration procedures will likely be most effective for solving various types of configuration problems (depending, in particular, on the number and type of target algorithm parameters, but also on regularities in the parameter response). Therefore, we see a need for research aiming to determine which configurator is most effective under which circumstances. In fact, we expect to find situations in which the sequential or iterative application of more than one configuration procedure turns out to be effective - for example, one could imagine applying FocusedILS to find promising configurations in vast, discrete configuration spaces, followed by a gradient-free numerical optimisation method, such as CMA-ES, for fine-tuning a small number of real-valued parameters. 
Overall, we believe that algorithm configuration techniques, such as the ones discussed in this chapter, will play an increasingly crucial role in the development, evaluation and use of state-of-the-art algorithms for challenging computational problems, where the challenge could arise from high computational complexity (in particular, $\mathscr{N} \mathscr{P}$-hardness) or from tight resource constraints (e.g., in realtime applications). Therefore, we see great value in the design and development of software frameworks that support the real-world application of various algorithm configuration and parameter tuning procedures. The High-Performance Algorithm Lab (HAL), recently introduced by Nell et al (2011), is a software environment designed to support a wide range of empirical analysis and design tasks encountered during the development, evaluation and application of high-performance algorithms for challenging computational problems, including algorithm configuration and parameter tuning. Environments such as HAL not only facilitate the application of automated algorithm configuration and parameter tuning procedures, but also their development, efficient implementation and empirical evaluation.

In closing, we note that the availability of powerful and effective algorithm configuration and parameter tuning procedures has a number of interesting consequences for the way in which high-performance algorithms are designed and used in practice. Firstly, for developers and end users, it is now possible to automatically optimise the performance of (highly) parameterised solvers specifically for certain classes of problem instances, leading to potentially much improved performance in real-world applications. Secondly, while on-line algorithm control mechanisms that adjust parameter settings during the run of a solver (as covered, for example, in Chapters ?? and ?? of this book) can in principle lead to better performance than the (static) algorithm configuration procedures considered in this chapter, we expect these latter procedures to be very useful in the context of (statically) configuring the parameters and heuristic components that determine the behaviour of these on-line control mechanisms. Finally, during algorithm development, it is no longer necessary (nor even desirable) to eliminate parameters and similar degrees of freedom, but instead, developers can focus more on developing ideas for realising certain heuristic mechanisms or components, while the precise instantiation can be left to automated configuration procedures (Hoos, 2008). We strongly believe that this last effect will lead to a fundamentally different and substantially more effective way of designing and implementing high-performance solvers for challenging computational problems.

\section{Acknowledgement}

This chapter surveys and discusses to a large extent work carried out by my research group at UBC, primarily involving Frank Hutter, Kevin Leyton-Brown and Kevin Murphy, as well as Thomas Stützle at Université Libre de Bruxelles, to all of whom I am deeply grateful for their fruitful and ongoing collaboration. I gratefully acknowledge valuable comments by Frank Hutter, Thomas Stützle and Maverick Chan on earlier drafts of this chapter, and I thank the members of my research 
group for the many intellectually stimulating discussions that provide a fertile ground for much of our work on automated algorithm configuration and other topics in empirical algorithmics.

\section{References}

Adenso-Diaz B, Laguna M (2006) Fine-tuning of algorithms using fractional experimental design and local search. Operations Research 54(1):99-114

Ansótegui C, Sellmann M, Tierney K (2009) A gender-based genetic algorithm for the automatic configuration of algorithms. In: Proceedings of the 15th International Conference on Principles and Practice of Constraint Programming (CP 2009), pp 142-157

Applegate DL, Bixby RE, Chvátal V, Cook WJ (2006) The Traveling Salesman Problem: A Computational Study. Princeton University Press

Audet C, Orban D (2006) Finding optimal algorithmic parameters using the mesh adaptive direct search algorithm. SIAM Journal on Optimization 17(3):642-664

Balaprakash P, Birattari M, Stützle T (2007) Improvement strategies for the f-race algorithm: Sampling design and iterative refinement. In: Bartz-Beielstein T, Blesa M, Blum C, Naujoks B, Roli A, Rudolph G, Sampels M (eds) 4th International Workshop on Hybrid Metaheuristics, Proceedings, HM 2007, Springer Verlag, Berlin, Germany, Lecture Notes in Computer Science, vol 4771, pp 108-122

Balaprakash P, Birattari M, Stützle T, Dorigo M (2010) Estimation-based metaheuristics for the probabilistic traveling salesman problem. Computers \& OR 37(11):1939-1951

Bartz-Beielstein T (2006) Experimental Research in Evolutionary Computation: The New Experimentalism. Natural Computing Series, Springer Verlag, Berlin, Germany

Bartz-Beielstein T, Lasarczyk C, Preuß M (2005) Sequential parameter optimization. In: McKay B, et al (eds) Proceedings 2005 Congress on Evolutionary Computation (CEC'05), Edinburgh, Scotland, IEEE Press, vol 1, pp 773-780

Bartz-Beielstein T, Lasarczyk C, Preuss M (2008) Sequential parameter optimization toolbox, manual version 0.5, September 2008, available at http://www.gm.fh-koeln.de/imperia/ $\mathrm{md} /$ content/personen/lehrende/bartz_beielstein_thomas/spotdoc.pdf

Battiti R, Brunato M, Mascia F (2008) Reactive Search and Intelligent Optimization. Operations Research/Computer Science Interfaces, Springer Verlag

Birattari M, Stützle T, Paquete L, Varrentrapp K (2002) A racing algorithm for configuring metaheuristics. In: GECCO '02: Proceedings of the Genetic and Evolutionary Computation Conference, pp 11-18

Birattari M, Yuan Z, Balaprakash P, Stützle T (2010) F-race and Iterated F-Race: An overview. In: Bartz-Beielstein T, Chiarandini M, Paquete L, Preuss M (eds) Experimental Methods for the Analysis of Optimization Algorithms, Springer, Berlin, Germany, pp 311-336

Búrmen Á, Puhan J, Tuma T (2006) Grid restrained Nelder-Mead algorithm. Computational Optimization and Applications 34(3):359-375

Carchrae T, Beck J (2005) Applying machine learning to low knowledge control of optimization algorithms. Computational Intelligence 21(4):373-387

Chiarandini M, Fawcett C, Hoos H (2008) A modular multiphase heuristic solver for post enrollment course timetabling (extended abstract). In: Proceedings of the 7th International Conference on the Practice and Theory of Automated Timetabling (PATAT 2008), 6 pages

Da Costa L, Fialho Á, Schoenauer M, Sebag M (2008) Adaptive Operator Selection with Dynamic Multi-Armed Bandits. In: Proceedings of the 10th Annual Conference on Genetic and Evolutionary Computation (GECCO’08), pp 913-920

Fawcett C, Hoos H, Chiarandini M (2009) An automatically configured modular algorithm for post enrollment course timetabling. Tech. Rep. TR-2009-15, University of British Columbia, Department of Computer Science 
Fukunaga AS (2002) Automated discovery of composite sat variable-selection heuristics. In: Proceedings of the 18th National Conference on Artificial Intelligence (AAAI-02), pp 641-648

Fukunaga AS (2004) Evolving local search heuristics for SAT using genetic programming. In: Genetic and Evolutionary Computation - GECCO-2004, Part II, Springer-Verlag, Seattle, WA, USA, Lecture Notes in Computer Science, vol 3103, pp 483-494

Gagliolo M, Schmidhuber J (2006) Dynamic algorithm portfolios. In: Amato C, Bernstein D, Zilberstein S (eds) Proceedings of the 9th International Symposium on Artificial Intelligence and Mathematics (AI-MATH-06)

Gomes CP, Selman B, Crato N, Kautz H (2000) Heavy-tailed phenomena in satisfiability and constraint satisfaction problems. Journal of Automated Reasoning 24(1-2):67-100

Gratch J, Chien SA (1996) Adaptive problem-solving for large-scale scheduling problems: A case study. Journal of Artificial Intelligence Research 4:365-396

Gratch J, Dejong G (1992) Composer: A probabilistic solution to the utility problem in speed-up learning. In: Rosenbloom P, Szolovits P (eds) Proceedings of the 10th National Conference on Artificial Intelligence (AAAI-92), AAAI Press / The MIT Press, Menlo Park, CA, USA, pp 235-240

Guerri A, Milano M (2004) Learning techniques for automatic algorithm portfolio selection. In: Proceedings of the 16th European Conference on Artificial Intelligence (ECAI 2004), pp 475479

Hansen N (2006) The CMA evolution strategy: a comparing review. In: Lozano J, Larranaga P, Inza I, Bengoetxea E (eds) Towards a new evolutionary computation. Advances on estimation of distribution algorithms, Springer, pp 75-102

Hansen N, Kern S (2004) Evaluating the CMA evolution strategy on multimodal test functions. In: Yao X, et al (eds) Parallel Problem Solving from Nature PPSN VIII, Springer, LNCS, vol 3242, pp 282-291

Hansen N, Ostermeier A (2001) Completely derandomized self-adaptation in evolution strategies. Evolutionary Computation 9(2):159-195

Hoos H (2008) Computer-aided design of high-performance algorithms. Tech. Rep. TR-2008-16, University of British Columbia, Department of Computer Science

Hoos H, Stützle T (2000) Local search algorithms for SAT: An empirical evaluation. Journal of Automated Reasoning 24(4):421-481

Hoos HH, Stützle T (2004) Stochastic Local Search-Foundations and Applications. Morgan Kaufmann Publishers, USA

Huang D, Allen TT, Notz WI, Zeng N (2006) Global optimization of stochastic black-box systems via sequential kriging meta-models. Journal of Global Optimization 34(3):441-466

Hutter F (2009) Automated configuration of algorithms for solving hard computational problems. $\mathrm{PhD}$ thesis, University of British Columbia, Department of Computer Science, Vancouver, BC, Canada

Hutter F, Tompkins DA, Hoos H (2002) Scaling and Probabilistic Smoothing: Efficient Dynamic Local Search for SAT. In: Principles and Practice of Constraint Programming - CP 2002, Springer-Verlag, LNCS, vol 2470, pp 233-248

Hutter F, Hamadi Y, Hoos HH, Leyton-Brown K (2006) Performance prediction and automated tuning of randomized and parametric algorithms. In: Principles and Practice of Constraint Programming - CP 2006, Springer-Verlag, LNCS, vol 4204, pp 213-228

Hutter F, Babić D, Hoos HH, Hu AJ (2007a) Boosting verification by automatic tuning of decision procedures. In: Proc. Formal Methods in Computer-Aided Design (FMCAD’07), IEEE Computer Society Press, pp 27-34

Hutter F, Hoos H, Stützle T (2007b) Automatic algorithm configuration based on local search. In: Proceedings of the 22nd National Conference on Artificial Intelligence (AAAI-07), pp 11521157

Hutter F, Hoos H, Leyton-Brown K, Murphy K (2009a) An experimental investigation of modelbased parameter optimisation: SPO and beyond. In: Proceedings of the 11th Annual Conference on Genetic and Evolutionary Computation (GECCO'09), ACM, pp 271-278 
Hutter F, Hoos H, Leyton-Brown K, Stützle T (2009b) ParamILS: An automatic algorithm configuration framework. Journal of Artificial Intelligence Research 36:267-306

Hutter F, Hoos H, Leyton-Brown K, Stützle T (2009c) ParamILS: An automatic algorithm configuration framework (extended version). Tech. Rep. TR-2009-01, University of British Columbia, Department of Computer Science

Hutter F, Hoos H, Leyton-Brown K (2010a) Sequential model-based optimization for general algorithm configuration (extended version). Tech. Rep. TR-2010-10, University of British Columbia, Department of Computer Science

Hutter F, Hoos H, Leyton-Brown K, Murphy K (2010b) Time-bounded sequential parameter optimization. In: Proceedings of the 4th International Conference on Learning and Intelligent Optimization (LION 4), Springer-Verlag, LNCS, vol 6073, pp 281-298

Hutter F, Hoos HH, Leyton-Brown K (2010c) Automated configuration of mixed integer programming solvers. In: Proceedings of the 7th International Conference on the Integration of AI and OR Techniques in Constraint Programming for Combinatorial Optimization Problems (CPAIOR 2010), Springer-Verlag, LNCS, vol 6140, pp 186-202

Hutter F, Hoos H, Leyton-Brown K (2011) Extending sequential model-based optimization to general algorithm configuration. To appear in: Proceedings of the 5th International Conference on Learning and Intelligent Optimization (LION 5)

Jones DR, Schonlau M, Welch WJ (1998) Efficient global optimization of expensive black box functions. Journal of Global Optimization 13:455-492

KhudaBukhsh A, Xu L, Hoos H, Leyton-Brown K (2009) SATenstein: Automatically building local search sat solvers from components. In: Proceedings of the 21 st International Joint Conference on Artificial Intelligence (IJCAI-09), pp 517-524

Leyton-Brown K, Nudelman E, Andrew G, McFadden J, Shoham Y (2003) A portfolio approach to algorithm selection. In: Rossi F (ed) Principles and Practice of Constraint Programming CP 2003, Springer Verlag, Berlin, Germany, Lecture Notes in Computer Science, vol 2833, pp 899-903

Lourenço HR, Martin O, Stützle T (2002) Iterated local search. In: Glover F, Kochenberger G (eds) Handbook of Metaheuristics, Kluwer Academic Publishers, Norwell, MA, USA, pp 321-353

Maron O, Moore AW (1994) Hoeffding races: Accelerating model selection search for classification and function approximation. In: In Advances in neural information processing systems 6 , Morgan Kaufmann, pp 59-66

Nelder JA, Mead R (1965) A simplex method for function minimization. The Computer Journal 7(4):308-313

Nell CW, Fawcett C, Hoos HH, Leyton-Brown K (2011) HAL: A framework for the automated design and analysis of high-performance algorithms. To appear in: Proceedings of the 5th International Conference on Learning and Intelligent Optimization (LION 5)

Nocedal J, Wright SJ (2006) Numerical Optimization, 2nd edn. Springer-Verlag

Nouyan S, Campo A, Dorigo M (2008) Path formation in a robot swarm: Self-organized strategies to find your way home. Swarm Intelligence 2(1):1-23

Pellegrini P, Birattari M (2006) The relevance of tuning the parameters of metaheuristics. a case study: The vehicle routing problem with stochastic demand. Tech. Rep. TR/IRIDIA/2006-008, IRIDIA, Université Libre de Bruxelles, Brussels, Belgium

Pop M, Salzberg SL, Shumway M (2002) Genome sequence assembly: Algorithms and issues. Computer 35(7):47-54

Prasad MR, Biere A, Gupta A (2005) A survey of recent advances in sat-based formal verification. International Journal on Software Tools for Technology Transfer 7(2):156-173

Rasmussen CE, Williams CKI (2006) Gaussian Processes for Machine Learning. The MIT Press

Rice JR (1976) The algorithm selection problem. Advances in Computers 15:65-118

Rossi-Doria O, Sampels M, Birattari M, Chiarandini M, Dorigo M, Gambardella LM, Knowles JD, Manfrin M, Mastrolilli M, Paechter B, Paquete L, Stützle T (2003) A comparison of the performance of different metaheuristics on the timetabling problem. In: Burke EK, Causmaecker PD (eds) Practice and Theory of Automated Timetabling IV, 4th International Conference, PATAT 
2002, Selected Revised Papers, Springer, Lecture Notes in Computer Science, vol 2740, pp 329-354

Sacks J, Welch W, Mitchell T, Wynn H (1989) Design and analysis of computer experiments (with discussion). Statistical Science 4:409-435

Santner T, Williams B, Notz W (2003) The Design and Analysis of Computer Experiments. Springer Verlag, New York

Schiavinotto T, Stützle T (2004) The linear ordering problem: Instances, search space analysis and algorithms. Journal of Mathematical Modelling and Algorithms 3(4):367-402

Schonlau M, Welch WJ, Jones DR (1998) Global versus local search in constrained optimization of computer models. In: Flournoy N, Rosenberger W, Wong W (eds) New Developments and Applications in Experimental Design, vol 34, Institute of Mathematical Statistics, Hayward, California, pp 11-25

Spall J (2003) Introduction to Stochastic Search and Optimization. John Wiley \& Sons, Inc., New York, NY, USA

Stützle T, Hoos HH (2000) MAX-MIN Ant System. Future Generation Computer Systems 16(8):889-914

Thachuk C, Shmygelska A, Hoos H (2007) A replica exchange monte carlo algorithm for protein folding in the hp model. BMC Bioinformatics 8(342)

Tompkins DA, Hoos HH (2010) Dynamic Scoring Functions with Variable Expressions: New SLS Methods for Solving SAT. In: Proceedings of the 13th International Conference on Theory and Applications of Satisfiability Testing (SAT 2010), Springer-Verlag, LNCS, vol 6175, pp 278292

Xu L, Hutter F, Hoos HH, Leyton-Brown K (2007) SATzilla-07: the design and analysis of an algorithm portfolio for SAT. In: Principles and Practice of Constraint Programming - CP 2007, Springer Berlin / Heidelberg, LNCS, vol 4741, pp 712-727

Xu L, Hutter F, Hoos HH, Leyton-Brown K (2008) SATzilla: portfolio-based algorithm selection for SAT. Journal of Artificial Intelligence Research 32:565-606

Xu L, Hutter F, Hoos H, Leyton-Brown K (2009) SATzilla2009: An Automatic Algorithm Portfolio for SAT, solver description, SAT Competition 2009

Xu L, Hoos H, Leyton-Brown K (2010) Hydra: Automatically configuring algorithms for portfoliobased selection. In: Proceedings of the 24th AAAI Conference on Artificial Intelligence (AAAI10), pp 210-216

Yuan Z, Fügenschuh A, Homfeld H, Balaprakash P, Stützle T, Schoch M (2008) Iterated greedy algorithms for a real-world cyclic train scheduling problem. In: Blesa M, Blum C, Cotta C, Fernndez A, Gallardo J, Roli A, Sampels M (eds) Hybrid Metaheuristics, Lecture Notes in Computer Science, vol 5296, Springer Berlin / Heidelberg, pp 102-116 